\title{
CARACTERIZAÇÃO E AVALIAÇÃO TEMPORAL DE REMANESCENTES FLORESTAIS DO MUNICÍPIO DE ARARAS/SÃO PAULO
}

\section{CHARACTERIZATION AND TEMPORAL EVALUATION OF FOREST REMNANTS IN THE CITY OF ARARAS, SÃO PAULO}

\section{Nathalia Fadel ${ }^{1}$; Olavo Raymundo Júnior²; Heitor Siqueira Sayeg $^{3}$}

'Bióloga, graduada pela Fundação Hermínio Ometto/UNIARARAS. E-mail: nathifadel@yahoo.com.br

${ }^{2}$ Doutor e pró-reitor de Graduação da Fundação Hermínio Ometto/UNIARARAS. Email: olavo@uniararas.br

${ }^{3}$ Engenheiro Geólogo, Instituto de Geografia da Universidade Federal de Uberlândia.

E-mail: hsayeg@hotmail.com

\begin{abstract}
RESUMO
O município de Araras/SP apresenta, aproximadamente, 5\% de seu território representado por remanescentes de vegetação nativa, os quais ainda são alvo de intervenções antrópicas. Este trabalho tem como objetivo diagnosticar os remanescentes florestais de Araras, por meio da análise temporal, no período entre 1962 e 2008 . As análises foram realizadas por meio de fotos aéreas datadas de 1962, 2000, e imagens de satélite CBERS/2008, nas escalas: 1:25.000, 1:30.000 e 1:20.000, respectivamente. O índice de circularidade foi utilizado como parâmetro para aferir a relação área/perímetro. Utilizou-se a classificação supervisionada da imagem para avaliar as informações sobre o efeito de borda. Por meio da análise temporal, pôde-se constatar que, nesse período, os fragmentos menores que 10 hectares correspondem a mais de $50 \%$ dos remanescentes de mata nativa. A utilização das Áreas de Preservação Permanente (APP's), algumas já florestadas e outras abandonadas, permitiria a formação de corredores ecológicos, unindo os fragmentos. $\mathrm{O}$ Índice de Circularidade demonstrou que 13,9\% dos fragmentos datados de 1962 e 7,4\% dos fragmentos analisados em imagens de 2000 apresentavam forma circular. Tanto nas imagens de 1962 como de 2000, a maior parte dos fragmentos apresentava forma alongada. Concluiu-se que, em 1962, o padrão de desmatamento em Araras/SP já estava definido, com 56\% dos fragmentos com área inferior a 10 hectares. Atualmente os fragmentos encontram-se impactados e isolados na paisagem. A recomposição das APP's poderia ser uma alternativa para diminuir o grau de isolamento. A análise temporal demonstrou que a área apresenta potencial de auto-regeneração.

Palavra chave: Diagnóstico ambiental. Geoprocessamento. Fragmentos florestais. Áreas de Preservação Permanente APP's.
\end{abstract}




\begin{abstract}
The Araras' City presents approximately 5\% of its territory represented by remnants of native vegetation, but still, are the target of anthropogenic interventions. This paper aims to diagnose the forests remnants in Araras, through temporal analysis in the periods between 1962, 2000 and 2008. The analyses were performed by means of aerial photos and satellite images CBERS/2008, preselected on scales: 1: 25,000, 1: 30,000 and 1: 20,000. The index of circularity was used as a parameter for measuring the relationship area/perimeter. It was used supervised image classification for evaluating the information on edge effect. Through temporal analysis, it can be seen that in this period the fragments smaller than 10 hectares correspond to more than $50 \%$ of native forest remnants. The use of the Permanent Preservation Areas (PPAs), some already afforested and other abandoned would allow the formation of ecological corridors, linking the fragments. The index of circularity demonstrated that $13.9 \%$ of fragments in 1962 and $7.4 \%$ in 2000 had rounded forms and most of them presented elongated form. It was concluded that in 1962, the pattern of deforestation in Araras, was already defined, with $56 \%$ of the fragments measuring less than 10 hectares. Currently the fragments are impacted and isolated in the landscape. The recomposition of PPA's could be an alternative to reduce the degree of isolation. The temporal analysis showed that the area has potential for self- regeneration.
\end{abstract}

Key words: Environmental diagnosis.Geoprocessing. Forest fragments. Permanent Preservation Areas (PPAs)

\title{
1. INTRODUÇÃO
}

A Mata Atlântica, nos séculos XVI e XVII, ocupava aproximadamente 15\% do atual território brasileiro ao longo da costa, constituindo um dos mais ricos biomas brasileiro. Todavia, através dos séculos, em épocas distintas, mas sempre crescente, foi submetida a diversos processos de intervenção antrópica, acarretando significativa redução de oferta de serviços ecológicos, gerando sérios impactos ecológicos negativos, especialmente a extinção de espécies (AGAREZ et al., 2001; FLEURY, 2003).

A atividade desordenada de uso e ocupação da terra, impulsionada pelo modelo econômico vigente e pelo crescimento populacional, tem resultado na degradação, fragmentação e esgotamento dos recursos florestais, nos quais a degradação está intimamente relacionada com a perda de hábitat. $\mathrm{O}$ aumento da relação perímetro/superfície das florestas é a consequência mais evidente da fragmentação florestal. Estudos ecológicos sobre projetos de restauração produzem informações importantes para compreensão de ecossistemas degradados e preservados (LAURANCE, 1994; RODRIGUES e LEITÃO-FILHO, 2000; NASCIMENTO et al., 2006).

As técnicas de sensoriamento remoto proporcionam uma visão espacial e temporal do processo de urbanização e permitem obter informações do meio ambiente urbano, das formas de uso do solo, e até sobre a inferência de determinadas características socioeconômicas (ALVES et al., 2009).

O município de Araras, SP apresenta 4,8\% de seu território com vegetação natural remanescente (KRONKA, 2005). Os estudos realizados em Araras (ALMEIDA et al., 2006; RAYMUNDO JR et al., 2007) demonstraram a importância de se aprofundar os estudos com enfoque regional para viabilizar ações práticas que possibilitem a conservação dos recursos naturais, que refletem diretamente na qualidade de vida da população.

O objetivo deste trabalho foi realizar o mapeamento e diagnóstico dos fragmentos de mata nativa no município de Araras, SP, por meio de análise temporal do processo de ocupação e estado de conservação no período compreendido entre os anos de 1962 a 2008 e identificar áreas que possam ser utilizadas como corredores ecológicos, visando à integração dos fragmentos. 


\section{MATERIAL E MÉTODOS}

\section{Descrição da área de estudo e atividades}

O município de Araras (Figura 1) está situado na região nordeste do estado de São Paulo, a $174 \mathrm{~km}$ da capital, com área de $643.46 \mathrm{~km}^{2}$, altitude de 650 metros e é um dos três municípios mais populosos da bacia do Rio Mogi-Guaçu (SÃO PAULO, 2009).

Segundo Oliveira et al. (1982), no levantamento pedológico do estado de São Paulo, quadrícula de Araras, observa-se estreita correspondência entre a qualidade do solo e seu aproveitamento. Entre os municípios Leme, Araras e sul de Conchal, sobre solos de textura argilosa derivados de rochas ígneas básicas e de sedimentos finos de idade permocarbonífera, a vegetação original, a julgar pelos reduzidos remanescentes, era representada principalmente pela mata subperenifólia. A vegetação natural é praticamente inexistente, substituída por agricultura em decorrência do relevo pouco acidentado e da qualidade das terras, sendo essas culturas constituídas principalmente de cana-de-açúcar, citros, algodão, café e, em menor proporção, por outras culturas, especialmente as anuais.

O cultivo da cana-de-açúcar representa $52,90 \%$, fruticultura $18,69 \%$, culturas anuais como milho, soja, mandioca $2,28 \%$, pastagens $2,08 \%$ e cafeicultura e silvicultura aproximadamente $1 \%$ da área total dos municípios (VALLADARES et al., 2008).

\section{Análise temporal}

$\mathrm{Na}$ caracterização e análise temporal da vegetação nativa e de APP's foram utilizadas fotos aéreas de 1962 e 2000, imagens do satélite brasileiro CBERS (China-Brazilian Earth Resources Satellite) ano 2008 e cartas topográficas da região de estudo (IBGE, 1969) na escala 1:50000. No "software" ARCGIS foram elaborados os mapas temáticos demarcando-se os fragmentos florestais, áreas de APP's e áreas abandonadas (áreas sem uso aparente) localizadas em torno dos corpos de água, por meio dos mosaicos compostos pelas fotos aéreas, respectivamente nas escalas 1:25.000 e 1:30.000. Foram realizados no "software" ERDAS Image o georreferenciamento, a composição do mosaico e a classificação supervisionada em imagens CBERS do ano de 2008 na escala 1:20.000. Essas informações foram todas ajustadas na escala de trabalho 1:10.000.

A partir dos mapas temáticos, foi realizada uma análise estatística utilizando como parâmetros: valores máximos e mínimos, as medidas de tendência central (média, mediana) e a medida de dispersão (desvio padrão).

A classificação supervisionada permitiu a identificação das classes de informação de interesse. Nestas, foram separadas as regiões de áreas já conhecidas que melhor representavam tais classes (MÁXIMO, 2005). Foram utilizados como parâmetros de pixel a Barragem Hermínio Ometto, uma área paludosa localizada à margem da Rodovia Conchal/Araras e os fragmentos de vegetação nativa localizados na Fazenda São José, entre os municípios de Araras e Rio Claro, descritos nos trabalhos de Pagano e Leitão Filho (1987) e Udulutsch, (2004).

A área foi descrita e estudada em visitas a campo e através de análises complementares baseadas em videografia em perspectiva em voos de baixa altitude (imagens inéditas do município capturadas em 2008 e 2010, cedidas pelo Dr. Heitor Siqueira Sayeg), além de interpretação das fotos aéreas.

Calculou-se a geometria dos fragmentos utilizando o índice proposto por Miller em 1953 (apud CHRISTOFOLETTI, 1974) no qual o cálculo da área (A), dividida pelo perímetro (P) vezes a constante $(\mathrm{K})$ fornece o índice de circularidade (IC - "Compactness ratio"), que determina a tendência de evolução do fragmento em relação à sua forma: 


$$
\begin{gathered}
\mathrm{IC}=\mathrm{K} .\left(\mathrm{A} / \mathrm{P}^{2}\right) \\
\mathrm{K}=12,57 \text { (constante) } \\
\mathrm{A}=\text { área do fragmento }\left(\mathrm{m}^{2}\right) \\
\mathrm{P}=\text { perímetro }(\mathrm{m})
\end{gathered}
$$

\section{RESULTADOS E DISCUSSÃO}

A análise dos fragmentos (Tabela 1) a partir das fotos aéreas do ano de 1962 revelou a existência de 130 fragmentos florestais que somaram 4.250,9 ha, o tamanho médio era de 42,5 ha com desvio padrão 73, a mediana obtida foi 9,01 ha. A maior área florestal apresentava 657,7 ha, e a menor 0,89 ha. Verificou-se que 56,5\% dos fragmentos eram inferiores a 10 ha, $30,6 \%$ estavam na faixa acima de 10 e 50 ha e $13 \%$ maiores que 50 ha. Nas fotos aéreas do ano 2000, foram mapeados 108 fragmentos, somando 3.261,3 ha de área total dos remanescentes da cobertura vegetal, com área média de 32,6 ha sendo o maior 575,9 ha e o menor 1,21 ha, o desvio padrão de 70 e a mediana de 9,22 ha. Constatou-se que 53,1\% dos fragmentos eram menores que 10 ha, $31,5 \%$ na faixa entre 10 e 50 ha e $15,4 \%$ maiores que 50 ha (Tabela 2). Os dados demonstraram que, tanto em 1962 como em 2000, mais de 50\% dos fragmentos tinham área menor que 10 ha, o que comprova que o padrão de desmatamento no município já estava definido desde 1962.

Tabela 1 - Comparação dos fragmentos florestais entre os anos de 1962 e 2000, por meio dos resultados obtidos pela análise de fotos aéreas.

\begin{tabular}{cccc}
\hline Fragmentos & $\mathbf{1 9 6 2 ( h a )}$ & $\mathbf{2 0 0 0 ( h a )}$ & Variação (2000-1962) \\
\hline $\mathbf{n}^{\mathbf{0}}$ de fragmentos & $\mathbf{1 3 0}$ & $\mathbf{1 0 8}$ & $\mathbf{- 2 2}$ \\
Área total & $4.250,90$ & $3.261,30$ & $-989,6$ \\
Área média & 42,5 & 32,6 & $-9,9$ \\
Maior área & 657,7 & 575,9 & $-81,8$ \\
Menor área & 0,89 & 1,21 & $+0,32$ \\
Mediana & 9,01 & 9,22 & $-0,21$ \\
Desvio padrão & 73 & 70 & -3 \\
(IC) médio & 0,45 & 0,36 & \\
\hline
\end{tabular}

Tabela 2 - Comparação do número de fragmentos entre os anos de 1962 e 2000.

\begin{tabular}{ccccc}
\hline Área - A (ha) & Ano 1962 & $\mathbf{( \% )}$ & Ano 2000 & $\mathbf{( \% )}$ \\
\hline $\mathrm{A} \leq 10$ & 69 & 53,1 & 61 & 56,5 \\
$10<\mathrm{A} \leq 50$ & 41 & 31,5 & 33 & 30,6 \\
$50<\mathrm{A}$ & 20 & 15,4 & 14 & 13 \\
$\Sigma$ & 130 & 100 & 108 & 100 \\
\hline
\end{tabular}


O município de Araras ocupa uma área de 64.346 ha. Considerando os resultados obtidos para o ano de 1962, quando o município apresentava 6,6\% de área de mata nativa em relação aqueles obtidos para o ano de 2000 , quando o município apresentava $5 \%$ de área de mata nativa, verifica-se redução de $32 \%$ da área florestada. Caso este processo de perda tenha sido gradual, visto que o padrão de ocupação do solo já estava definido em 1962, estima-se a perda de $8,42 \%$ de área de mata nativa a cada 10 anos.

Estudo realizado por Greggio et al. (2009), entre os anos de 1971 e 2000, avaliando a evolução temporal e o número de fragmentos florestais no período de 29 anos no município de Jaboticabal,SP, verificou-se a diminuição da cobertura vegetal. Esses autores demonstraram que esta estava fortemente fragmentada, apresentando apenas 3,63\% da área do município com vegetação em 1971 e 1,51\% em 2000, sendo a taxa de perda de vegetação semelhante àquela encontrada neste estudo (Figura 1).

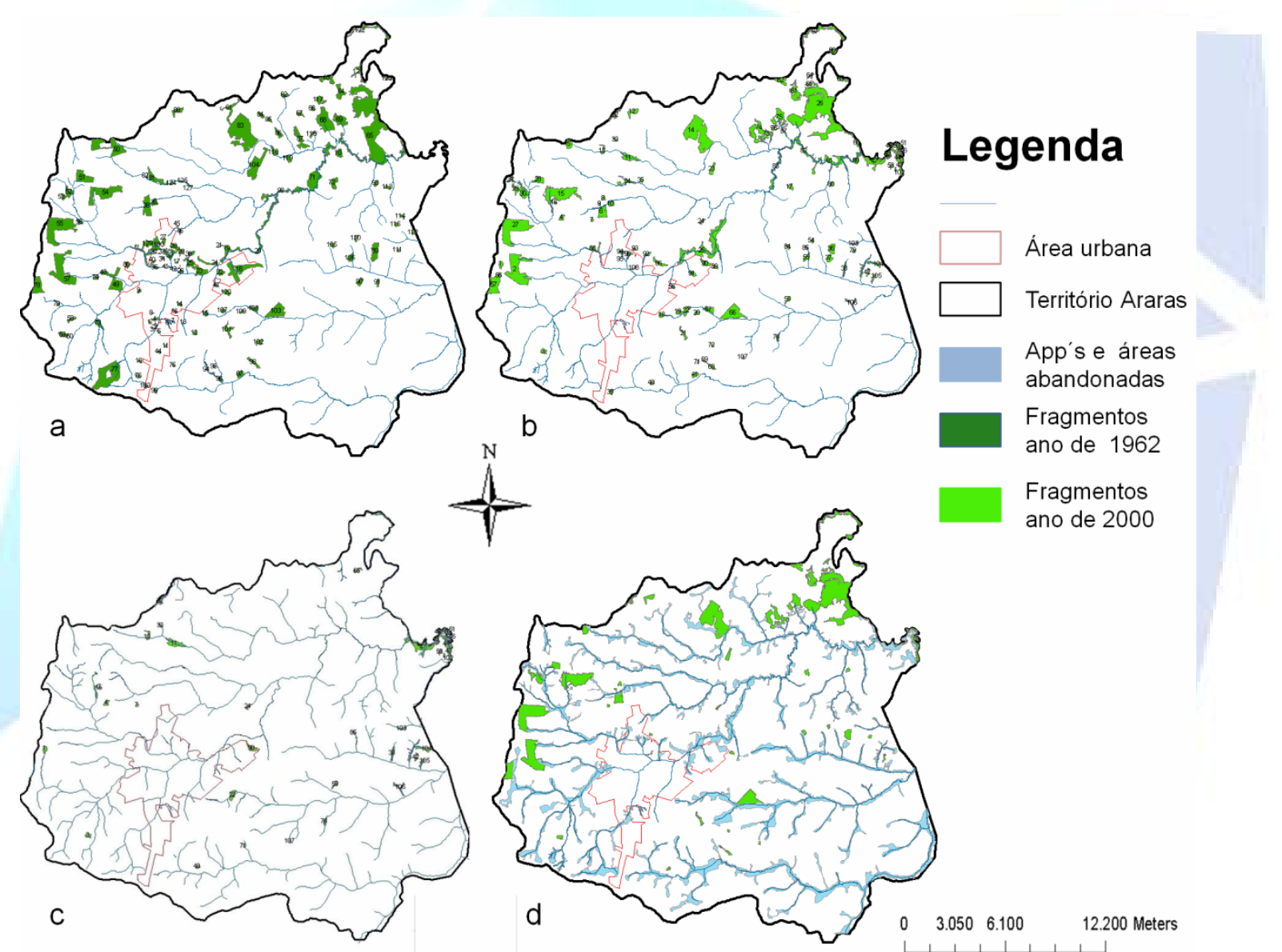

Figura 1. Fragmentos Florestais do município de Araras: a) ano 1962; b) ano 2000; c) fragmentos que apareceram no ano de 2000; e d) áreas de APP's ou abandonadas nas margens dos corpos de água que podem ser utilizadas como corredores ecológicos.

Entre os anos de 1962 e 2000 constatou-se que 42 fragmentos se mantiveram, porém com alteração de suas formas e seus estados de conservação, de acordo com os índices de circularidade calculados; 74 fragmentos desapareceram durante o período; três fragmentos se fundiram, demonstrando que a área manteve o potencial de regeneração (Figura $1 \mathrm{a}, \mathrm{b}$ ).

A análise das fotos aéreas de 2000 (Figura 1c) mostra 40 fragmentos inexistentes em 1962, que passaram a figurar na paisagem. Os maiores fragmentos que se regeneraram estavam associados 
a rios ou corpos de água, indicando haver um processo de regeneração natural dessas áreas, visto que não há registro de recomposição de áreas naturais no município antes do ano 2000. Este fato é importante, pois, apesar do município apresentar-se com baixíssima taxa de cobertura vegetal (5\%), há um potencial de regeneração observado.

Segundo Viana e Pinheiro (1998), a conectividade entre os fragmentos florestais tende a diminuir em paisagens mais intensamente cultivadas. Podem ser identificadas diversas estratégias para o aumento da conectividade entre os fragmentos, destacando-se o estabelecimento de corredores em matas ciliares e encostas. A escolha da estratégia mais apropriada para um determinado fragmento depende de análise de custo-benefício caso a caso.

Uma vez que o município de Araras apresenta rede hídrica extensa e com grande necessidade de recomposição, foram identificadas nas áreas de APP's extensões abandonadas no entorno de corpos de água (Figura 1d). Essas áreas, além de proteger os mananciais e ajudar a garantir o abastecimento de água do município, também poderiam compor corredores ecológicos, promovendo a conectividade entre os fragmentos florestais. Dessa forma, verificou-se que seria possível unir todos os fragmentos restantes por meio da recomposição das APP's do município.

Segundo Santos et al. (2009), o fator área dos fragmentos é muito relevante, pois ao se calcular a média destes em diferentes áreas, considera-se que os maiores fragmentos vão ter regeneração muito mais significativa do que os menores, ou seja, nos primeiros será encontrada maior diferenciação de fatores que influenciará a formação da vegetação como, por exemplo, variação da altitude, variação das formas do relevo, maior diferenciação da insolação em diferentes localidades de um mesmo fragmento, entre outros.

$\mathrm{O}$ índice de circularidade (IC) classificou os fragmentos observados no município de Araras até $\mathrm{IC}=0,70$ (alongado) e acima de $\mathrm{IC}=0,70$ (circular). A média dos fragmentos em 1962 foi de $\mathrm{IC}=$ 0,45 e em 2000 de IC $=0,36$.

Conforme descrito na Tabela 3, o (IC) foi ordenado em classes para permitir a melhor discussão dos dados. Os fragmentos com tendência à forma circular (o valor de IC é próximo de um '1') apresentam menor razão borda/interior. Quanto mais este valor se distancia de um '1', mais o fragmento é alongado, com razão borda/interior progressivamente mais alta. Neste contexto, a primeira situação é a desejável com a menor razão borda/interior possível (BORGES et al., 2004; MARTINS et al., 2009).

Tabela 3 - Classificação dos fragmentos florestais no município de Araras, SP, conforme o Índice de Circularidade (IC), nos anos de 1962 e 2000.

\begin{tabular}{ccccc}
\hline Classes IC & \multicolumn{3}{c}{ Fragmentos florestais } \\
\hline & \multicolumn{3}{c}{1962} & \multicolumn{2}{c}{2000} \\
& № de frag. & (\%) & № de frag. & (\%) \\
\hline 0,1 a 0,5 & 74 & 56,9 & 78 & 72,2 \\
0,5 a 0,6 & 19 & 14,6 & 11 & 10,2 \\
0,6 a 0,7 & 19 & 14,6 & 11 & 10,2 \\
0,7 a 0,8 & 14 & 10,8 & 7 & 6,5 \\
0,8 a 1,0 & 4 & 3,1 & 1 & 0,9 \\
\hline
\end{tabular}

As Tabelas 2 e 3 mostram que no intervalo compreendido entre 1962 e 2000, houve diminuição no número total de fragmentos, de 130 para 108, com decréscimo do tamanho médio de 42,5 para 32,6 ha, somado a alteração no padrão da fragmentação das áreas, com incremento de 74 para 78 fragmentos com IC $<0,5$, um salto de $56,9 \%$ para $72,2 \%$ do total de fragmentos, considerando-se também que nas faixas superiores a $\mathrm{IC}>0,5$ deu-se a diminuição do número 
destes, isto demonstrando a fragilidade dos mesmos quanto a resistência do efeito de borda, devido ao aumento no processo de fragmentação.

Fragmentos com tendência à forma circular apresentam uma 'área core' que é definida por Aziz Ab'Saber (2003), como sendo:

[...] domínios espaciais de feições paisagísticas e ecológicas integradas, ocorrem em uma espécie de área principal, de certa dimensão e arranjo, em que as condições fisiográficas e biogeográficas, formam um complexo relativamente homogêneo e extensivo. A essa área mais típica e contínua - via de regra, de arranjo poligonal - aplicamos o nome de área core, logo traduzida por área nuclear [...] (AB'SABER, 2003)

Nos anos de 1962 e 2000 os IC dos fragmentos foram, respectivamente, de 56,9\% e 72,2\% na classe de $0,1-0,5 ; 14,6 \%$ e $10,2 \%$ para a classe de $0,5-0,6 ; 14,6 \%$ e $10,2 \%$ para a classe de 0,6 $-0,7 ; 10,8 \%$ e $6,5 \%$ para a classe de $0,7-0,8 ; 3,1 \%$ e $0,9 \%$ com IC acima de 0,8 .

Os estudos realizados na região norte do Paraná por Paula e Rodrigues (2002) mostraram que os fragmentos com áreas maiores tendem a ser mais alongados. No fato da maioria dos fragmentos florestais que apresentam IC próximo de 1 resistir mais ao efeito de borda, deve-se levar em conta que naqueles com áreas diminutas o efeito de borda é mais pronunciado. Segundo Viana e Pinheiro (1998), o fator forma de matas ciliares é relativamente pouco considerado para que estas apresentem regularmente forma alongada.

Com base nestas informações acima citadas, constatou-se que muitos dos fragmentos classificados com forma circular apresentavam área relativamente baixa, o que se torna preocupante, pois pequenos fragmentos tendem a desaparecer ou se descaracterizar. Embora muitos fragmentos florestais, identificados neste estudo, apresentem área bastante reduzida, torna-se importante evidenciar que a sua conservação contribui para a manutenção ou o aumento da diversidade biológica local. (NASCIMENTO et al., 2006).

A classificação supervisionada foi realizada a fim de conferir os mapas gerados por meio das fotos aéreas e para atualização da geometria dos fragmentos, esta confronta os mapas temáticos gerados a partir das fotos aéreas (Figura 2). Neste estudo, constataram-se dois padrões de pixels nos fragmentos escolhidos para classificação supervisionada, um deles relacionado a um padrão de vegetação mais adensada, com o dossel estruturado, denominado mata A, (Figura 2).

O segundo padrão correspondeu a um dossel desestruturado, com grande presença de lianas e espécies invasoras, denominado mata B. Estas descrições tiveram como base a checagem em campo e imagens obtidas por videografia em voo de baixa altitude. De acordo com Engel et al. (1998) em florestas alteradas e em fragmentos florestais, geralmente a abundância de cipós aumenta muito, podendo atingir níveis nos quais os mecanismos de autorregulação ou homeostase do ecossistema ficam comprometidos.

Pagano e Leitão Filho (1987) descreveram os fragmentos que foram escolhidos para a classificação supervisionada, como sendo de floresta. A mesma localiza-se em solo fértil e com variações de fertilidade, que possibilita a ocorrência de um considerável número de espécies com o estrato superior. Este representado pelas famílias Leguminosae, Euphorbiaceae, Lauraceae, Anacardiaceae e Arecaceae e o estrato inferior, representado pelas famílias Rutaceae, Meliaceae, Euphorbiaceae e Rubiaceae, subarbustivas e herbáceas. Apresentam também clareiras, áreas ribeirinhas de pequenos cursos deágua e áreas em diversos estados de sucessão existentes no interior da mata, com grande presença de trepadeiras nas bordas e nas clareiras. 


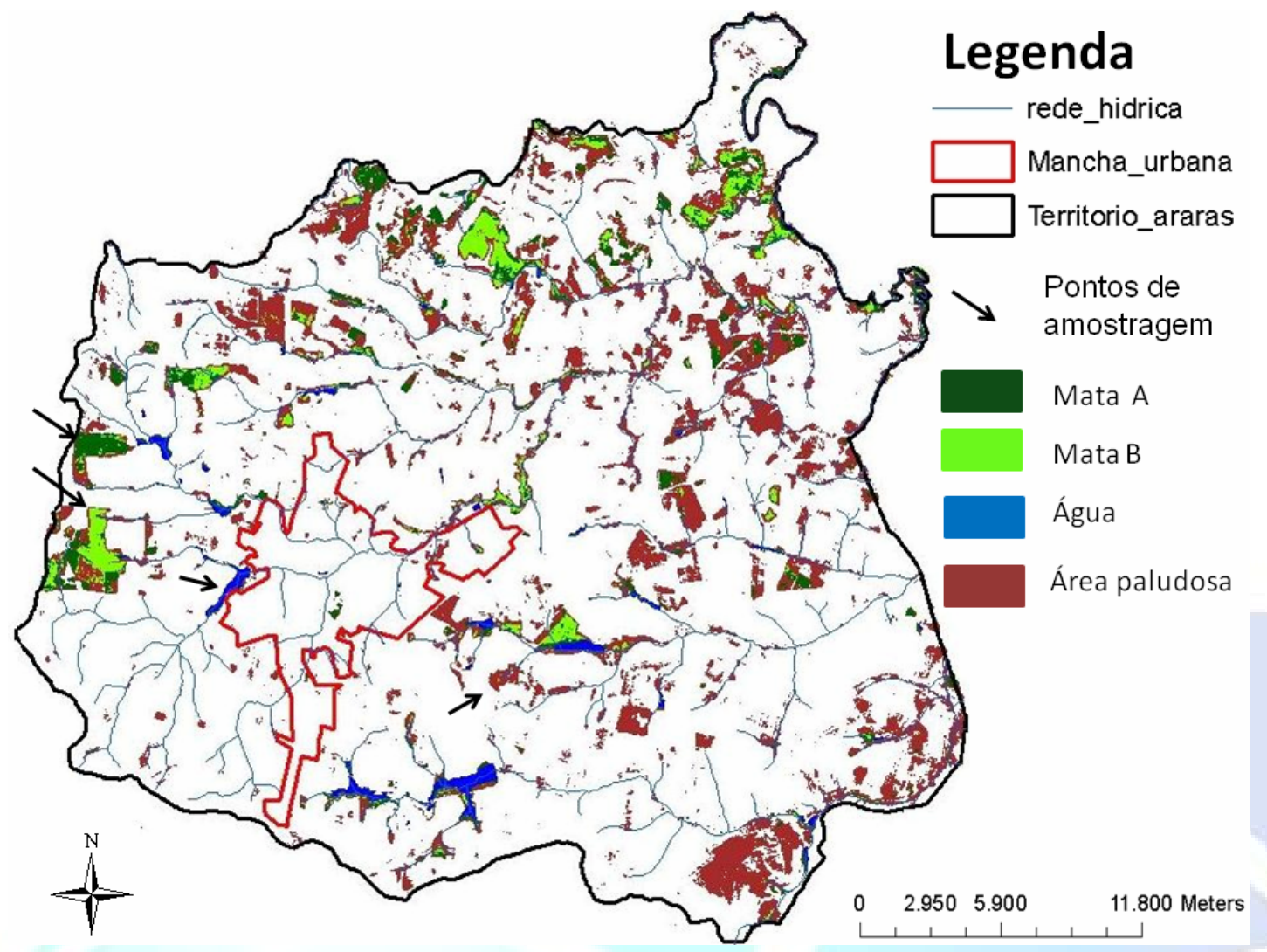

Figura 2. Classificação supervisionada do município de Araras - São Paulo: destacam-se os pontos marcados por setas, locais de amostragem de pixel para a confecção da Classificação Supervisionada da imagem.

Na Figura 2 foram inseridas a rede hídrica e outras áreas identificadas na classificação supervisionada, além dos fragmentos. Destacaram-se também as represas e áreas paludosas, com afloramento do lençol freático. Muitas das áreas paludosas na checagem em campo estavam ocupadas por plantações de laranja e cana-de-açúcar.

\section{CONCLUSÕES}

O município de Araras se encontra fortemente impactado pelas atividades agrícolas. $\mathrm{O}$ padrão de desmatamento já estava estabelecido em 1962, com 56\% dos fragmentos com área inferior a 10 ha. Identificou-se potencial de regeneração natural, pois fragmentos que não existiam em 1962 passaram a figurar em 2000.

As APP's podem servir como corredores ecológicos, o que permitiria a ligação de todos os fragmentos observados.

A classificação supervisionada identificou dois padrões de pixels para a vegetação nativa, que estão associados a diferentes padrões de dossel, predominando as matas com dossel desestruturado, impactadas pelo efeito de borda.

\section{AGRADECIMENTOS}

Os autores agradecem ao Centro Universitário Hermínio Ometto - FHO/UNIARARAS e ao Conselho Nacional de Desenvolvimento Científico e Tecnológico - CNPq pelo apoio financeiro. 


\section{REFERÊNCIAS}

AB'SABER, A.N. Os domínios de natureza no Brasil: potencialidades paisagísticas. São Paulo: Ateliê Editorial, 2003. 159p.

AGAREZ, F.V.; VICENS, R.S.; CRUZ, C.B.M.; NOGUEIRA, C.R.; GARAY, I. Utilização de Índice de vegetação na classificação integrada de fragmentos florestais em Mata Atlântica de Tabuleiros no município de Sooretama, ES. In: X Simpósio Brasileiro de Sensoriamento Remoto, 2001, Foz do Iguaçú. Anais... São José dos Campos: INPE, 2001. p. 1-9.

ALVES, C.D.; PEREIRA, M.N.; FLORENZANO, T.G. Mapeamento das novas formas de ocupação urbana por meio da análise orientada a objeto. Estudo de caso: Aglomeração Urbana de Piracicaba. In: XIV Simpósio Brasileiro de Sensoriamento Remoto Anais... Natal, Brasil, 25-30 abril 2009, INPE, p. 569-576.

ALMEIDA, T.C.; BISNETO, R.T.; SAYEG, H.S.; RAYMUNDO JR, O. Diagnóstico preliminar das condições ambientais da área de manancial do município de Araras/SP.. In: 19. RAIB -

Reunião Anual do Instituto Biológico, 2006, São Paulo. Biológico, 2006. v. 68. p. 1-4.

BORGES, L.F.R.; SCOLFORO, J.R.; OLIVEIRA, A.D.; MELLO, J.M.; ACERBI, F.W.; FREITAS, G.D. Inventário de Fragmentos Florestais Nativos e Propostas para seu Manejo e o da Paisagem. Cerne, Lavras-MG, v.10, n.1, p. 22-38, jan./jun. 2004.

CHRISTOFOLETTI, A. Geomorfologia. Ed. Edgard Blucher Ltda e EDUSP. 1974. 149 p.

FLEURY, M. Efeito da Fragmentação florestal e na predação de sementes da palmeira Jerivá (Syagrus romanzoffiana) em florestas semidecíduas do estado de São Paulo. São Paulo. 2003, 101f. Dissertação (Mestrado) - Escola Superior de Agricultura "Luis de Queiróz" Universidade de São Paulo, Piracicaba. 2003.

ENGEL, V.L.; FONSECA, R.C.B.; OLIVEIRA, R.E. Ecologia de Lianas e o manejo de fragmentos florestais. Série Técnica IPEF, Piracicaba-SP, v. 12, n. 32, p. 43-64, dez. 1998.

GREGGIO, T.C.; PISSARA, T.C.; RODRIGUES, F.M. Avaliação dos fragmentos florestais do município de jaboticabal-sp. Árvore, Viçosa-MG, v.33, n.1, p.117-124, 2009.

KRONKA, F.J.N.; NALON, M.A.; MATSUKUMA, C.K.; KANASHIRO, M.M.; YWANE, M.S.S.; PAVÃO, M.; DURIGAN, G.; LIMA, L.M.P.R.; GUILLAUMON, J.R.; BAITELLO, J.B.; BORGO, S.C.; MANETTI, L.A.; BARRADAS, A.M.F.; FUKUDA, J.C.; SHIDA, C.N.; MONTEIRO, C.H.B.; PONTINHA, A.A.S.; ANDRADE, G.G.; BARBOSA, O; SOARES, A.P.; COUTO, H. T. Z. do; JOLY, C. A Inventário florestal da vegetação natural do Estado de São Paulo. São Paulo: Instituto Florestal, Imprensa Oficial, 2005. 200p.

IBGE. INSTITUTO BRASILEIRO DE GEOGRAFIA E ESTATÍSTICA. Carta topográfica da folha de Araras (SP). Rio de Janeiro: Fundação IBGE. 1 mapa. Escala 1:50.000. 1969.

LAURANCE, W.F. Rainforest fragmentation and the structure of a small mammal comunites in tropical Queensland. Conservation Biology, n. 69, p. 23-32. 1994. 
MARTINS, I.C.M.; SOARES,V.P.; SILVA, E.; BRITES, R.S. Caracterização ambiental de fragmentos florestais naturais - "ipucas"- no município de Lagoa da Confusão, Tocantins. R. Árvore, Viçosa-MG, v. 26, n.3, p 299-309, 2002.

MÁXIMO, O.A.; FERNANDES, D. Classificação supervisionada de imagens SAR do SIVAM préfiltradas. In: XII Simpósio Brasileiro de Sensoriamento Remoto Anais..., Goiânia, Brasil, 16-21 abril 2005, INPE, p. 4139-4146.

NASCIMENTO, M.C.; SOARES, V.P.; RIBEIRO, C.A. A. S.; SILVA, E. Mapeamento dos Fragmentos de Vegetação Florestal Nativa da Bacia Hidrográfica do Rio Alegre, Espírito Santo, a partir de Imagens do Satélite Ikonos II. Árvore, Viçosa-MG, v.30, n.3, p.389-398, 2006.

OLIVEIRA, J.B.; MENK, J.R.F.; BARBIERI, J.L.; ROTTA, C.L.; TREMACOLDI, W. Levantamento pedológico semidetalhado do Estado de São Paulo. Quadrícula de Araras. Secretaria de Agricultura e Abastecimento, Instituto Agronômico.Campinas-SP, p.1-180, 1982.

PAGANO, S.N.; LEITÃO FILHO, H.F.Composição floristica do estrato arbóreo de mata mesófila semidecídua, no município de Rio Claro (Estado de São Paulo). Revista Brasileira de Botânica, v.10, n.1, p.37-47, 1987.

PAULA, A.S.; RODRIGUES, E. Degradação da paisagem norte-paranaense: um estudo de fragmentos florestais Semina: Ciências Agrárias, Londrina-PR, v. 23, n. 2, p. 229-238, jul./dez. 2002

RAYMUNDO JR, O. ; ALMEIDA, T.C.; SAYEG, H.S.; BISNETO, R.T.; MENDES, J.A. Uso e ocupação do solo na microbacia do Ribeirão de Furnas em área de manancial no município de Araras, SP. OLAM, Rio Claro-SP, v. 7, p. 262-275, 2007.

RODRIGUES, R.R., LEITÃO FILHO, H.F. Matas ciliares: conservação e recuperação. São Paulo: Edusp-Fapesp. 2000. 320p.

SANTOS, R.H.L.; MACHADO, D.C.S., CRUZ, C.B.M.; SEABRA, V.S.; GONÇALVES, E.S. Análise dos níveis de degradação da floresta através da utilização do NDVI. In: XIV Simpósio Brasileiro de Sensoriamento Remoto Anais... Natal, Brasil, 25-30 abril 2009, INPE, p. 3011-3018.

SÃO PAULO. SECRETARIA DO MEIO AMBIENTE. Coordenadoria de recursos Hídricos. Situação dos Recursos Hídricos do Estado de São Paulo. São Paulo, 2009.

UDULUTSCH, R.G.; ASSIS, M.A.; PICCHI, D.G. Florística de trepadeiras numa floresta estacional semidecídua, Rio Claro - Araras, Estado de São Paulo, Brasil. Revista Brasileira de Botânica, São Paulo, v. 27, n. 1, p.125-134, 2004.

VALLADARES, G.S.; AVANCINI, C. S.A.; TÔSTO, S.G. Uso e Cobertura das terras do município de Araras. Circular Técnica on-line Embrapa, Campinas, SP, n. 14, p. 1-12, 2008.

VIANA, V.M.; PINHEIRO, L.A.F.V. Conservação da biodiversidade em fragmentos florestais . Série Técnica IPEF, Piracicaba - SP, v.12, n.32, p.25-42, 1998. 